\title{
Are we getting to lipid targets in real life?
}

Niki Katsiki ${ }^{1,2}$, Dimitri P. Mikhailidis ${ }^{1}$, Vasilis G. Athyros ${ }^{3}$, Apostolos I. Hatzitolios ${ }^{2}$, Asterios Karagiannis ${ }^{3}$, Maciej Banach ${ }^{4}$ 1Department of Clinical Biochemistry (Vascular Disease Prevention Clinics), Royal Free
Hospital Campus, University College London Medical School, University College London,
London, United Kingdom
${ }^{21} 1^{\text {st }}$ Propedeutic Department of Internal Medicine, AHEPA University Hospital, Aristotle
University of Thessaloniki, Thessaloniki, Greece
${ }^{3} 2^{\text {nd }}$ Propedeutic Department of Internal Medicine, Hippokration Hospital, Medical School,
Aristotle University of Thessaloniki, Thessaloniki, Greece
${ }^{4}$ Department of Hypertension, Chair of Nephrology and Hypertension, Medical University
of Lodz, Lodz, Poland

Submitted: 27 August 2010

Accepted: 4 September 2010

Arch Med Sci 2010; 6, 5: 639-641

DOI: 10.5114/aoms.2010.17073

Copyright @ 2010 Termedia \& Banach

In this issue of Archives of Medical Science a Hungarian group reports on lipid goal achievement in 12,317 high risk patients [1]. This analysis considers the treatment of dyslipidemia between 2004 and 2008 and also compares the performance of general practitioners (GPs) and specialists (9,508 and 2,809 patients, respectively). During this period the percentage of patients "on target" for low density lipoprotein-cholesterol (LDL-C) increased from $14 \%$ to $32 \%$ and $20 \%$ to $43 \%$ when treated by GPs and specialists, respectively. In contrast, there was no significant improvement in high density lipoprotein-cholesterol (HDL-C) or triglyceride (TG) levels. The conclusion was that "more attention needs to be paid to changing treatment of patients to achieve target levels". This includes an increased use of combination therapy.

The authors [1] attribute the improvement in lipid management between 2004 and 2008 to the application of up-to-date consensus recommendations. More specifically, information was provided to physicians via journals, scientific meetings and country-level coordinators.

We previously conducted 4 pilot best-practice implementation enhancement programmes aimed at improving risk factor control [2-5]. Physicians from Hospitals or Health Centres recruited patients with metabolic syndrome (MetS) (628 patients, the SAGE-METS [Standardized arrangement for a guideline driven treatment of the metabolic syndrome] study) [2], diabetes mellitus (DM) (578 patients, the INDEED [Initiative for a new Diabetes therapeutic approach in a Mediterranean country] study) [3], hypertension (697 patients, the IMPULSION [Implementation of guidelines for the management of arterial hypertension] study) [4] or dyslipidemia (1127 patients, the IMPROVE-dyslipidemia [Implementation of strategy for the management of overt dyslipidemia] study) [5].

Participating physicians attended educational programmes related to current guidelines for MetS, DM, hypertension, dyslipidemia, overt cardiovascular disease (CVD), obesity and nutrition [2-5]. The physicians were motivated to participate as part of their continuing medical education program. Patients were also motivated by supplying them with a brochure that included instructions to help them achieve treatment goals.

\section{Corresponding author:}

Dimitri P. Mikhailidis

Department of Clinical

Biochemistry (Vascular Disease

Prevention Clinics)

Royal Free Hospital Campus

University College London

Medical School

University College London

London, United Kingdom

E-mail: mikhailidis @ad.com 
The prevalence of dyslipidemia was reduced from 79 to $24 \%$ in the SAGE-METS study [2], from 76 to $12 \%$ in the INDEED study [3], from 59.7 to $53.6 \%$ in the IMPULSION study [4] and from 100 to $21 \%$ in the IMPROVE-dyslipidemia study [5]. The greatest reduction in LDL-C levels (31.6\%) was observed in patients with dyslipidemia (IMPROVEdyslipidemia study) [5]. Triglyceride levels were significantly reduced in all patient populations ( $p$ from $<0.002$ to $<0.0001$ for all comparisons). High density lipoprotein-cholesterol levels increased in all studies $(p<0.0001)$ except for the IMPULSION study where the rise was not significant.

All these studies [1-5] highlight the importance of continuous education of medical practitioners. Mark et al. [1] reported a higher percentage of patients achieving LDL-C targets when treated by specialists compared with those followed up by GPs ( $43 \%$ vs. $32 \%$, respectively; $p<0.0001$ by our calculations). In our studies [2-5] the performance of specialists and GPs was not compared.

Mark et al. [1] suggested that the high percentage of Hungarian patients on a statin may be at least partly attributed to available generic agents which are more affordable. The impact of this effect is likely to increase as more statins (and other lipidlowering drugs) become generic. These authors [1] also specified that the use of combination therapy (e.g. statin plus ezetimibe) contributed to better goal achievement. This interpretation is in agreement with community-based studies that showed a significantly improved outcome in lipid targets following the addition of ezetimibe to a statin [6, 7]. Overall, a meta-analysis $(n=5,039)$ concluded that ezetimibe co-administration with a statin provides significant additional lipid-lowering effect (a further 23.6\% reduction in LDL-C levels; $p<0.0001)$, allowing more patients to achieve LDL-C target values [8]. Similar results were obtained in another recent meta-analysis [9]. Higher doses of statins represent another therapeutic option, although this may be associated with an increased risk of adverse effects [10].

Although reduction of LDL-C remains the primary goal for lipid-lowering interventions, other targets (e.g. HDL-C and TG) may also be important [11-13]. In this context, non-HDL-C has also been proposed as a marker of residual CVD risk $[14,15]$. Another potential therapeutic goal would be apolipoprotein (apo) A and B levels or their ratio [14, 16-18]. The advantage when using non-HDL-C, apo A and/or apo $B$ is that these markers can be measured in the nonfasting state $[14,16,17]$. However, there is no definitive evidence showing that raising HDL-C levels in patients on statins will result in a significant reduction in vascular events $[19,20]$. Similarly, it is not absolutely clear if TG levels (fasting or non-fasting) are independent predictors of vascular risk [17, 21-24]; however TG, besides LDL-C and C-reactive protein (CRP), seems to be predictors of cardiovascular events especially in patients with acute coronary syndromes [12, 25].

The role of "over the counter (OTC)" statins [26, 27] in achieving LDL-C goals is controversial [28-30]. Limitation of OTC statins include the fact that many countries have not approved this process, the dose allowed to be prescribed by the pharmacist is low (e.g. $10 \mathrm{mg}$ simvastatin in the UK) and the cost has to be paid by the patient $[26,27]$.

Finally, we must deal with all vascular risk factors (e.g. hypertension, smoking, DM, obesity) and not just lipids [31, 32]. We should also keep in mind the increased CVD risk of certain populations (e.g. South Asians) and the possibility of different therapeutic targets in those patients [33, 34].

\section{References}

1. Mark L, Paragh G, Karadi I, et al. Changes in attaining lipid goals by general practitioners and specialists in patients at high cardiovascular risk in Hungary between 20042008. Arch Med Sci 2010; 6: 695-700.

2. Athyros VG, Karagiannis A, Hatzitolios Al, et al. Standardized arrangement for a guideline-driven treatment of the metabolic syndrome: the SAGE-METS study. Curr Med Res Opin 2009; 25: 971-80.

3. Athyros VG, Hatzitolios Al, Karagiannis A, et al. Initiative for a new diabetes therapeutic approach in a Mediterranean country: the INDEED study. Curr Med Res Opin 2009; 25: 1931-40.

4. Karagiannis A, Hatzitolios Al, Athyros VG, et al. Implementation of guidelines for the management of arterial hypertension. The impulsion study. Open Cardiovasc Med J 2009; 3: 26-34.

5. Hatzitolios Al, Athyros VG, Karagiannis A, et al. Implementation of strategy for the management of overt dyslipidemia: the IMPROVE-dyslipidemia study. Int J Cardiol 2009; 134: 322-9.

6. Fras Z, Mikhailidis DP. Statin plus ezetimibe treatment in clinical practice: the SI-SPECT (Slovenia (SI) Statin Plus Ezetimibe in Cholesterol Treatment) monitoring of clinical practice study. Curr Med Res Opin 2008; 24: 2467-76.

7. Migdalis I, Efthimiadis A, Pappas S, et al. Clinical experience with ezetimibe/simvastatin in a Mediterranean population. Curr Med Res Opin 2009; 25: 2571-6.

8. Mikhailidis DP, Sibbring GC, Ballantyne CM, et al. Metaanalysis of the cholesterol-lowering effect of ezetimibe added to ongoing statin therapy. Curr Med Res Opin 2007; 23: 2009-26.

9. Angelopoulos J, Krassakopoulos N, Nathanson R, Boukas $\mathrm{S}$, Sampalis JS. Co-administration of ezetimibe and a statin in management of dyslipidemias: a meta-analysis of clinical trials. Arch Med Sci 2009; 5: 347-63.

10. Kiortsis DN, Filippatos TD, Mikhailidis DP, et al. Statinassociated adverse effects beyond muscle and liver toxicity. Atherosclerosis 2007; 195: 7-16.

11. Athyros VG, Mikhailidis DP, Kakafika Al, et al. Identifying and attaining LDL-C goals: mission accomplished? Next target: new therapeutic options to raise HDL-C levels. Curr Drug Targets 2007; 8: 483-8.

12. Wainwright G, Mascitelli L, Goldstein MR. Cholesterollowering therapy and cell membranes. Stable plaque at 
the expense of unstable membranes? Arch Med Sci 2009; 5: 289-95.

13. Tziomalos K, Athyors VG, Karagiannis A, et al. Triglycerides and vascular risk: insights from epidemiological data and interventional studies. Curr Drug Targets 2009; 10: 320-7.

14. Brunzell JD, Davidson M, Furberg C, et al. Lipoprotein management in patients with cardiometabolic risk. Consensus statement from the American Diabetes Association and the American College of Cardiology Foundation. Diabetes Care 2008; 31: 811-22.

15. Brewer HB Jr. New features of the National Cholesterol Education Program Adult Treatment Panel III lipid-lowering guidelines. Clin Cardiol 2003; 26 (Suppl. 3): III19-24.

16. McQueen MJ, Hawken S, Wang X, et al; INTERHEART study investigators. Lipids, lipoproteins anf apolipoproteins as risk markers of myocardial infarction in 52 countries (the INTERHEART study): a case-control study. Lancet 2008; 372: 224-33.

17. The Emerging Risk Factors Collaboration. Major lipids, apolipoproteins and risk of vascular disease. JAMA 2009; 302: 1993-2000.

18. Sniderman A, Solhpour A. Targeting targets for LDL-lowering therapy: lessons from the Collaborative Atorvastatin Diabetes Study (CARDS). Clin Chem 2009; 55: 391-3.

19. Briel M, Ferreira-Gonzalez I, You JJ, et al. Association between changes in high density lipoprotein cholesterol and cardiovascular disease morbidity and mortality: systematic review and meta-regression analysis. BMJ 2009; 338: b92.doi: 10.1136/bmj.b92

20. Ridker PM, Genest J, Boekholdt SM, et al; JUPITER Trial Study Group. HDL cholesterol and residual risk of first cardiovascular events after treatment with potent statin therapy: an analysis from the Jupiter trial. Lancet 2010; 376: 333-9.

21. Triglyceride Coronary Disease Genetics Consortium and Emerging Risk Factors Collaboration. Triglyceride-mediated pathways and coronary disease: collaborative analysis of 101 studies. Lancet 2010; 375: 1634-9.

22. Sarwar N, Danesh J, Eiriksdottir G, et al. Triglycerides and the risk of coronary heart disease: 10,158 incident cases among 262,525 participants in 29 Western prospective studies. Circulation 2007; 115: 450-8.

23. Harchaoui KE, Visser ME, Kastelein JJ, et al. Triglycerides and cardiovascular risk. Curr Cardiol Rev 2009; 5: 216-22.

24. Kolovou G, Anagnostopoulou K, Mikhailidis DP. One century of triglycerides, but there is still lots to learn! Curr Drug Targets 2009; 10: 299-301.

25. Banach M, Mikhailidis DP, Kjeldsen SE, Rysz J. Time for new indications for statins? Med Sci Monit 2009; 15: MS1-5.

26. MHRA 2004. Reclassification summary for simvastatin POM to P. Medicines and Healthcare Products Regulatory Agency at http:// medicines.mhra_gov.uk.

27. Royal Pharmaceutical Society of Great Britain, 2004 Practice guidance on sale of over-the-counter simvastatin [online]. Available: http:// www.rpsgb.org.uk/pdfs/ otcsimvastatinguid.pdf

28. Tinetti ME. Over-the-Counter Sales of Statins and Other Drugs for Asymptomatic Conditions. New Engl J Med 2008; 358: 2728-32

29. Brass EP, Vassil T, Replogle A, et al. Can consumers selfselect for appropriate use of an over-the-counter statin? The Self Evaluation of Lovastatin to Enhance Cholesterol Treatment Study. Am J Cardiol 2008; 101: 1448-50.

30. Gemmel I, Verma A, Harrison RA. Should we encourage over-the-counter statins? A population perspective for coronary heart disease prevention. Am J Cardiovasc Drugs 2007; 7: 299-302.
31. Landini L, Leone A, Mikhailidis DP. Modifying cardiovascular risk factors: newer insights and preventive measures. Curr Pharm Des 2009; 15: 1034-7.

32. Desai RV, Banach M, Ahmed MI, et al. Impact of baseline systolic blood pressure on long-term outcomes in patients with advanced chronic systolic heart failure (insights from the BEST trial). Am J Cardiol 2010; 106: 221-7.

33. Dreiher J, Cohen A, Weitzman S, Sharf A, Shvartzman P. Lipid levels among African and Middle-Eastern Bedouin populations. Med Sci Monit 2008; 14: CR339-344.

34. Tziomalos K, Weerasinghe CN, Mikhailidis DP, Seifalian AM. Vascular risk factors in South Asians. Int J Cardiol 2008; 128: 5-16. 